\title{
Osteomyelitis of the pubic symphysis caused by methicillin-resistant Staphylococcus aureus after vaginal delivery: a case report and literature review
}

\author{
Stefano Cosma ${ }^{1}$, Fulvio Borella ${ }^{1 *}$ (D), Andrea Carosso ${ }^{1}$, Agata Ingala² Federica Fassio², Tiziana Robba ${ }^{3}$, Aldo Maina ${ }^{4}$,
} Luca Bertero ${ }^{5}$ and Chiara Benedetto ${ }^{1}$

\begin{abstract}
Background: Osteomyelitis of the pubic symphysis is a rare cause of pelvic pain after delivery, mainly caused by Staphylococcus aureus and Pseudomonas aeruginosa.

The clinical context is the same as the more common diastasis of the pubic bone, but the presence of intense local pain in association with fever should prompt further clinical work-up based on blood chemistry, microbiology and diagnostic imaging. We report the first case of methicillin-resistant Staphylococcus aureus osteomyelitis of the pubic symphysis occuring after the delivery.

Case presentation: A 39-year-old woman developed pain over the pubic bone $12 \mathrm{~h}$ after the delivery. After $72 \mathrm{~h}$ fever rose and laboratory examination showed elevation of C-reactive protein and procalcitonin levels. Pelvic x-rays and magnetic resonance showed pubic diastasis, joint effusion, tiny irregularities of articular surfaces and, severe bone edema. The patient was started on broad spectrum intravenous (IV) antibiotics (piperacillin-tazobactam) and then replaced to IV vancomycin and oral levofloxacin based on antibiogram result. She was then discharged with oral antibiotic therapy and fully recovered.

Conclusions: Due to the rarity of this disease, we compared our experience with the other cases of osteomyelitis of pubic symphysis occurring in peri-postpartum reported in the literature. The course of osteomyelitis was favourable in all patients, and only in one case an additional orthopedic procedure for symphysis fixation was necessary. Knowledge of this rare condition is important to enable prompt diagnosis and treatment.
\end{abstract}

Keywords: Pregnancy, Postpartum, Pubic diastasis, Septic arthritis, Osteomyelitis, Staphylococcus aureus

\section{Background}

Osteomyelitis of the pubic symphysis is a rare infectious disease associated with local bone destruction and frequent involvement of the joint (septic arthritis).

This infection is commonly caused by the opportunistic bacteria Staphylococcus aureus (S. aureus) and Pseudomonas aeruginosa ( $P$. aeruginosa). Typical clinical features are pubic and groin pain, pubic tenderness, fever $>38^{\circ} \mathrm{C}$, leukocytes $>11 \times 10^{9} / \mathrm{L}$, and bacteremia. The main risk

\footnotetext{
* Correspondence: fulvio.borella87@gmail.com

${ }^{1}$ Gynecology and Obstetrics 1, Department of Surgical Sciences, City of

Health and Science, University of Torino, Torino, Italy

Full list of author information is available at the end of the article
}

factors are previous female incontinence surgery, intense physical activity, pelvic malignancy, and intravenous drugs abuse, however, septic arthritis of the pubic symphysis may exceptionally occur as a complication of the postpartum [1-3]. Persistent postpartum pubic pain is quite common and may be caused by diastasis of pubic bone with the widening of the symphysis from 4 to $5 \mathrm{~mm}$ (normal value) up to $5 \mathrm{~cm}$. The peripartum pubic separation occurs from 1 in 300 to 1 in 30,000 deliveries [4]. Septic arthritis of the same site is an extremely rare cause of postpartum pelvic pain and only a few cases have been reported in the medical literature so far [5-11]. We report the case of a 39-year-old woman who developed an

(c) The Author(s). 2019 Open Access This article is distributed under the terms of the Creative Commons Attribution 4.0 International License (http://creativecommons.org/licenses/by/4.0/), which permits unrestricted use, distribution, and reproduction in any medium, provided you give appropriate credit to the original author(s) and the source, provide a link to the Creative Commons license, and indicate if changes were made. The Creative Commons Public Domain Dedication waiver (http://creativecommons.org/publicdomain/zero/1.0/) applies to the data made available in this article, unless otherwise stated. 
osteomyelitis and septic arthritis of pubic symphysis caused by methicillin-resistant $S$. aureus (MRSA) after a normal vaginal delivery and compare our experience with other cases previously reported. Informed, written consent was received from the patient for publication.

\section{Case presentation}

A 39-year-old pregnanat (para 3) at 41 weeks of gestation was admitted to Sant'Anna Hospital with active labor. Her clinical history was notable for gestational diabetes mellitus. At 35 weeks of gestation, the patient performed a vaginal swab for group B streptococcus screening, and was negative. She delivered a macrosomic fetus of $4530 \mathrm{~g}$ without complications during the labor.

Twelve hours later she complained of increasing pain over the pubic bone radiating to the groin. She could not stand or walk and any active or passive movement of thighs and hips (flexion/extension and adduction/abduction) evoked intense regional pain. Her symptoms were thought to be related to pelvic girdle strain during delivery. After $72 \mathrm{~h}$ her temperature rose to $39.5 \mathrm{C}^{\circ}$, while her clinical examination was otherwise unremarkable. Laboratory investigation revealed leukocytosis $\left(21 \times 10^{9} / \mathrm{L}\right.$, reference range [ref] $4-10 \times 10^{9} / \mathrm{L} ; 85.3 \%$ neutrophils) and elevation of C-reactive protein (CRP) levels $(379.0 \mathrm{mg} / \mathrm{L}$, ref. $5.0 \mathrm{mg} / \mathrm{L})$ raised the suspicion of an undetected infection. Her serum procalcitonin (PCT) was elevated $(4.0 \mathrm{ng} / \mathrm{mL}$, ref.: negative $<0.5 \mathrm{ng} / \mathrm{ml}$, gray zone $0.5-2.0 \mathrm{ng} / \mathrm{ml}$, positive $>2.0 \mathrm{ng} / \mathrm{ml}$ ). Blood culture was performed and the patient was started on broad spectrum intravenous (IV) antibiotics (piperacillin-tazobactam $4.5 \mathrm{~g}$ every $6 \mathrm{~h}$ ). At the same time, transperineal ultrasound evaluation showed a round-shaped hypoechogenic, fluid-filled cavity between the articular surfaces of the pubis. At day 4 a plain X-ray showed a pubic diastasis of $2 \mathrm{~cm}$ and tiny irregularities of articular surfaces (Fig. 1). The blood cultures were positive for Gram-positive cocci, later identified as MRSA, so the antibiotic regimen was changed to IV vancomycin $(1 \mathrm{~g}$ twice daily) and oral levofloxacin (500 $\mathrm{mg}$ once a day), this choice was based on the result of antibiogram. A screening for MRSA was also performed on the newborn and was negative. At day 8, the patient underwent a pelvic magnetic resonance imaging (MRI) showing symphysis enlargement, abundant joint effusion with synovial thickening forming a pseudo-capsulated fluid collection within the symphysis, severe bone edema involving both pubic branches and edematous subcutaneous tissues (Fig. 2). These clinical and radiological findings were highly suggestive of acute osteomyelitis and septic arthritis. The patient improved after 2 weeks and then IV vancomycin was stopped. She was discharged after 18 days of hospitalization and continued oral levofloxacin (500 $\mathrm{mg}$ gr once a day) plus rifampicin $(600 \mathrm{mg}$ once a day). Finally, the antibiotic therapy was stopped after 4 weeks; the patient was afebrile with normal blood tests and she did not report local pubic pain or functional limitations. Nine months after delivery the patient was still asymptomatic and pelvic MRI revealed complete fluid reabsorption at the pubic symphysis.

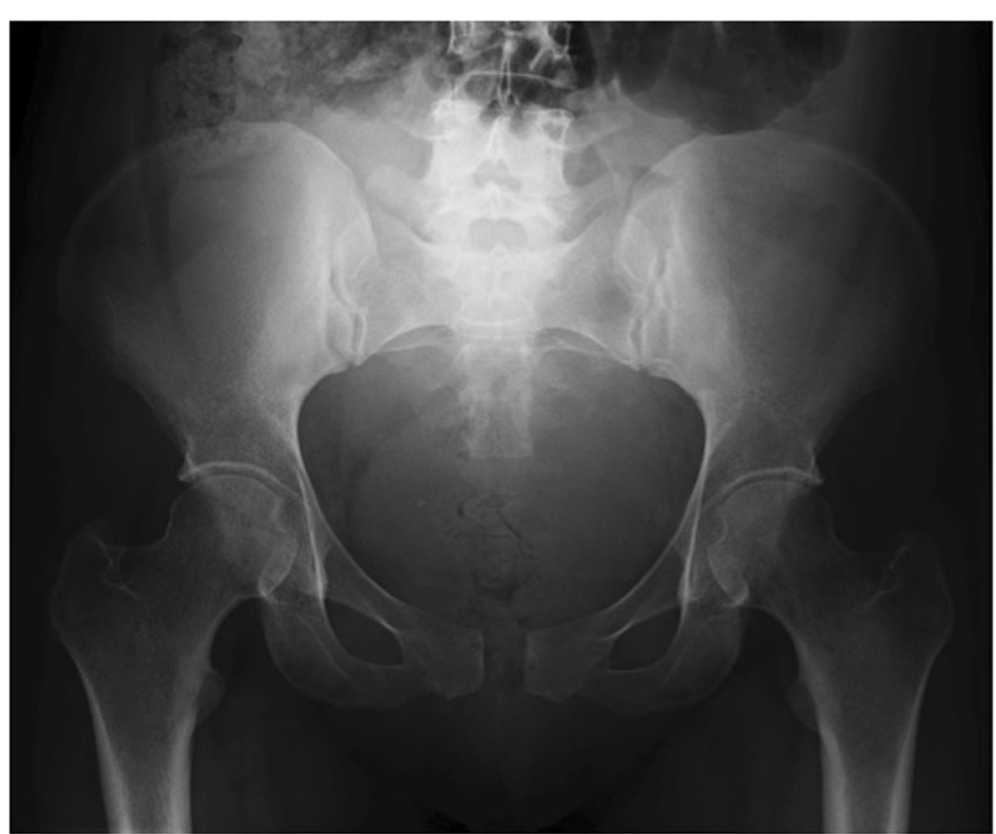

Fig. 1 Plain X-ray of the pelvis performed at day 4. The antero-posterior pelvic radiograph reveals pubic symphysis diastasis and tiny irregularities of articular surfaces 


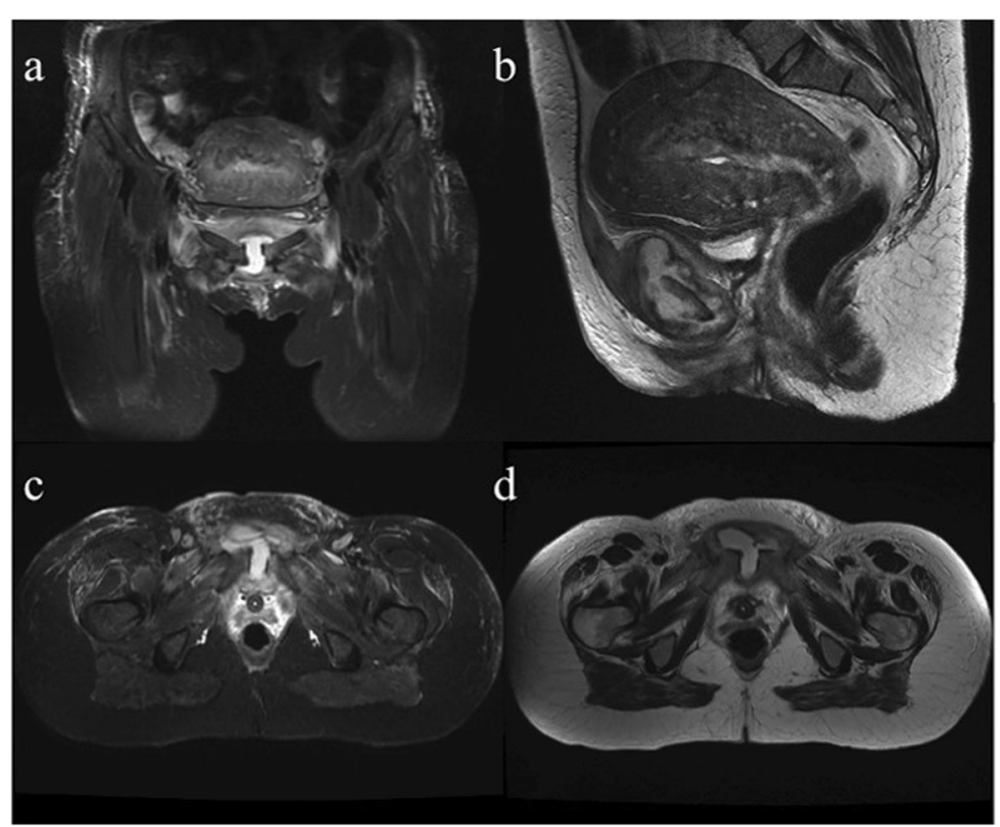

Fig. 2 Pelvic magnetic resonance performed at day 8. Coronal short TI inversion recovery (STIR) (a), sagittal T2-weighted (b), axial STIR (c), and axial T2-weighted (d) images show pubic symphysis enlargement, abundant joint effusion with synovial thickening forming a pseudo-capsulated fluid collection within the symphysis, severe bone edema involving both pubic branches and edematous subcutaneous tissues

\section{Discussion and conclusions}

A literature search was conducted using PubMed, EMBASE and Google Scholar. For this purpose, we used the following key words "osteomyelitis pubic symphysis", "septic arthritis pubic symphysis", "pubic symphysitis", "pubic osteitis", "pregnancy" "postpartum" and "delivery". With the inclusion of our patient, only 9 cases of osteomyelitis of the pubic symphysis in peri/postpartum have been reported in the literature and detailed information regarding these cases are reported in Table 1 [5-11].

No particular obstetric risk factors were reported for this disease: most cases were preceded by normal vaginal delivery. Only in one case the delivery was complicated by shoulder dystocia [5] and in two cases the osteomyelitis of pubic symphysis occurred after cesarean section $[7,10]$. The pathogenesis of this rare peripartum complication is unknown. In one case, the onset of pubic pain was concurrent with submandibular cellulitis and the authors suggested a hematogenous origin of the pubic infection [5].

The most common pathogens involved in osteomyelitis were $S$. aureus and $P$. aeurginosa, a trend further confirmed by our case of osteomyelitis of pubic symphysis [6$8,10]$. Other pathogens reported in peri/postpartum were Serratia marcescens, Streptococcus group G, Cutibacterium acnes, Staphylococcus capitis, Staphylococcus epidermidis and Enterococcus faecalis [5-11]. In our and previously reported cases of osteomyelitis of pubic symphysis, the culture tests were always positive for a pathogen while in 4 cases the total blood count was normal $[7,8,10,11]$.
All patients were treated with antibiotic therapy and only in 5 cases a surgical debridement was performed [5-9]. No patient had sequelae after the treatment and only in one case stabilization of the pubic symphysis by an external frame was required [6]. Also in our case, antibiotic therapy alone was sufficient to achive a successful outcome.

Postpartum pubic pain is a common and self-limiting disorder requiring only symptomatic therapy in most cases. The distinction between the more common diastasis and osteomyelitis of the pubic symphysis is critical for prognosis and treatment; moreover, non-infective pubis osteitis can occur after delivery, which can cause mild fever and has similar X-ray findings to those of osteomyelitis creating a potential diagnostic pitfall [2]. Local pain, inability to walk and fever are suggestive of an inflammatory/infective process, but the clinical suspicion should be confirmed by laboratory data, blood and/ or local fluid cultures and imaging findings.

Puerperal endometritis, characterised by pelvic pain, uterine or parametrial tenderness, maternal tachycardia, foulsmelling lochia or maternal leukocytosis $\left(>12 \times 10^{9} / \mathrm{L}\right)$, should also be considered in the differential diagnosis [12]. Regarding laboratory tests, in acute staphylococcal osteomyelitis, about $40 \%$ of patients presented with only a moderate leukocytosis count of $\left(10.5 \times 10^{9} / \mathrm{L}\right.$ considering a range of 4.5 to $11 \times 10^{9} / \mathrm{L}$ ) and in up to $40 \%$ of cases, microbiological tests produced false-negative results [3]. In 4 out of the 9 reported osteomyelitis of pubic symphysis 


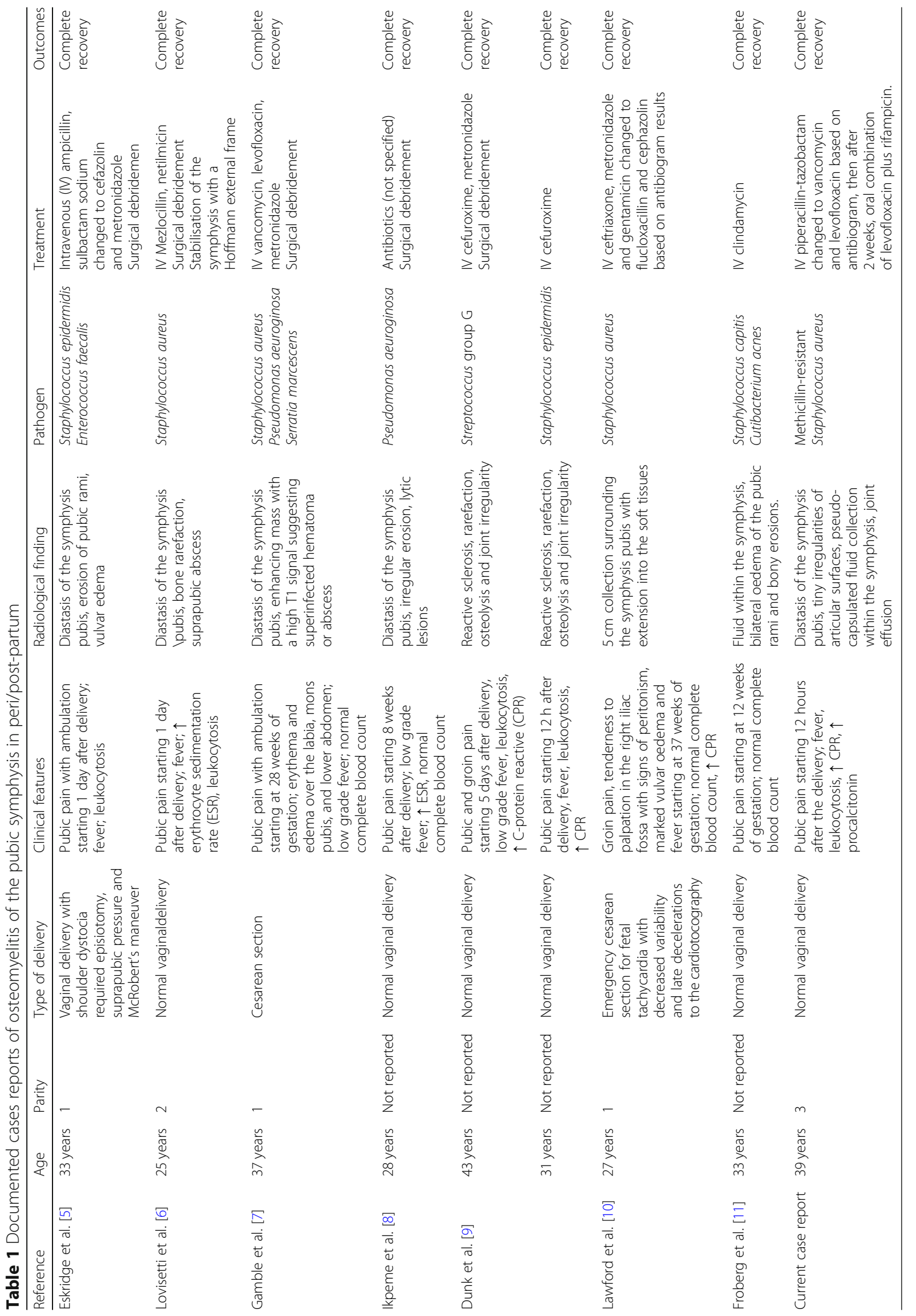


cases, a normal blood count was observed, but culture tests resulted consistently positive.

Possibly, in predisposed subjects, the pelvic soft tissues trauma occurring during vaginal delivery or cesarean section can promote the colonization and the contiguous spread of some bacterial strains, moreover, the colonization of the genital tract in pregnancy by MSRA, with the risk of vertical transmission, has been described in the literature [13, 14].

In one case, the onset of pubic pain was concurrent with submandibular cellulitis suggest a hematogenous origin of the pubic infection [5].

Of note, some authors $[15,16]$ also suggest to perform a screening of high-risk newborns for MRSA colonization or infection in order to promptly implement preventive measures.

Determination of serum PCT had been reported in a large series of osteomyelitis/septic arthritis, however the value often falls within a" gray zone" and false-negative results do occur, suggesting the use of a lower cut-off value to define positivity in this setting [17], however this marker may be more valuable than CRP for the diagnosis of this infectious disease [18], Paccolat et al evaluated PCT levels in pregnant women with no signs of clinical infection at different gestational ages; the authors suggest using a cut-off PCT level of $0.25 \mathrm{ng} / \mathrm{mL}$ to rule out infection during the third trimester, at delivery, and at the immediate postpartum [19], this is the first case of osteomyelitis of pubic symphysis in postpartum in which the PCT proved to be an useful infection marker.

Diagnostic imaging plays a crucial role in providing a correct framework for differential diagnosis of pubic pain. Transperineal or suprapubic sonography may be useful to reveal symphysis diastasis and show fluid collections, also enabling an ultrasound-guided needle aspiration for fluid aspiration or open bone biopsy with histopathologic examination and culture which are the gold standard for the microbiologic diagnosis of osteomyelitis. In our case, fluid aspiration was deferred because blood cultures were positive and consistent with radiologic findings. X-ray allows detecting symphysis diastasis, juxta-articular osteoporosis and bony erosions, but only in an advanced stage. Delayed signs of septic arthritis could be sclerosis, osteophytosis, and progressive joint destruction. The same abnormalities may be identified through computed tomography achieving a better evaluation of bony erosions, surface irregularities, abscesses and symphyseal diastasis [20]. However, magnetic resonance is the most sensitive tool and it allows early detection of suspicious findings for septic arthritis such as synovial enhancement, perisynovial edema, and joint effusion. Specificity, however, is not high since it is difficult to distinguish between reactive edema and osteomyelitis [20]. MRI also enables the identification of other possible causes of pubic pain due to postpartum complications such as pelvic hematoma or abscess, genitourinary injuries, and insufficiency fractures [21]. Furthermore, MRI findings are helpful to assess the response to treatments, especially the amount of joint effusion and the presence of abscess are probably the most reliable factors for monitoring therapy efficacy in osteomyelitis/septic arthritis [22].

Based on the reported cases, outcome seems to be excellent in most cases if prompt treatment is established. Regarding the specific regimen for MRSA osteomyelitis, optimal duration of antibiotic therapy is still a topic of debate: the clinical guidelines by the Infectious Disease Society of America for the treatment of MRSA infection suggest a minimum of 8 weeks for osteomyelitis and a 3-4-week of therapy for septhic arthritis, but a precise duration of the antibiotic therapy has not been clearly defined [3, 23, 24].

In conclusion, postpartum osteomyelitis of pubic symphysis is a rare disorder, which should be considered in women presenting with increasing pubic pain that does not respond to painkillers. Broad spectrum antibiotics are the first line treatment and must be administered while culture tests are ongoing. Surgical debridement may be considered in case of non-response to antibiotics; nevertheless, in staphylococcal osteomyelitis, relapse can occur in up to $40 \%$ of cases after surgical debridement [3]. Knowledge of this rare possible complication is necessary to enable prompt diagnosis and timely treatment.

\section{Abbreviations \\ CRP: C-reactive protein; ESR: Erythrocyte sedimentation rate; IV: Intravenous; MRI: Magnetic resonance imaging; MRSA: Methicillin-resistant S. aureus; PCT: Procalcitonin; Ref: Reference range}

\section{Acknowledgements}

We would like to thank Dr. Russo Arianna for the valuable suggestions to improve the manuscript.

\section{Authors' contributions}

Conception and design: SC, FB, CB. Acquisition of data: AC, Al, FF, TR, AM. Interpretation of data: All authors. Drafting the article: SC, FB, LB. Critically revising the article: $S C, F B, L B, C B$. Literature search: $A C, A I, F F, T R, A M$. Administrative/technical/material support: AC, Al, FF, TR, AM. Study supervision: SC, CB. All authors approved the final version of the manuscript.

\section{Funding}

No funding was sought or secured in relation to this case report.

\section{Availability of data and materials}

All data analysed during this study are included in this published article.

\section{Ethics approval and consent to participate}

This a retrospective case report, the ethical approval was not required due to the retrospective nature of the study, as stated by our institutional review board.

\section{Consent for publication}

Written informed consent for publication of their clinical details and/or clinical images was obtained from the patient. 


\section{Competing interests}

The authors declare that they have no competing interests.

\section{Author details}

${ }^{1}$ Gynecology and Obstetrics 1, Department of Surgical Sciences, City of Health and Science, University of Torino, Torino, Italy. ${ }^{2}$ Gynecology and Obstetrics 2, Department of Surgical Sciences, City of Health and Science, University of Torino, Torino, Italy. ${ }^{3}$ Department of Radiology, City of Health and Science, CTO Hospital, Torino, Italy. ${ }^{4}$ General Medicine Unit, City of Health and Science, Sant'Anna Hospital, Torino, Italy. ${ }^{5}$ Department of Medical Sciences, City of Health and Science, University of Torino, Torino, Italy.

Received: 16 August 2019 Accepted: 24 October 2019

Published online: 08 November 2019

\section{References}

1. Ross JJ, Hu LT. Septic arthritis of the pubic symphysis: review of 100 cases. Medicine. 2003:82(5):340-5.

2. Knoeller SM, Uhl M, Herget GW. Osteitis or osteomyelitis of the pubis? A diagnostic and therapeutic challenge: report of 9 cases and review of the literature. Acta Orthop Belg. 2006;72:541-8.

3. Kavanagh N, Ryan EJ, Widaa A, Sexton G, Fennell J, O'Rourke S, et al. Staphylococcal osteomyelitis: disease progression, treatment challenges, and future directions. Clin Microbiol Rev. 2018;14(2):31.

4. Jayaraman JK, Prabha G, Nalliannan I. Postpartum diastasis of the pubic Symphysis: report of a rare case. J Clin Diagn Res. 2015;9(9):QD09-10.

5. Eskridge C, Longo S, Kwark J, Robichaux A, Begneaud W. Osteomyelitis pubis occurring after spontaneous vaginal delivery: a case presentation. J Perinatol. 1997;17(4):321-4.

6. Lovisetti G, Sala F, Battaini A, Lovisetti L, Guicciardi E. Osteomyelitis of the pubic symphysis, abscess and late disjunction after delivery. A case report. Chir Organi Mov. 2000;85(1):85-8.

7. Gamble K, Dardarian TS, Finstein J, Fox E, Sehdev H, Randall TC. Osteomyelitis of the pubic symphysis in pregnancy. Obstet Gynecol. 2006; 107:477-81.

8. Ikpeme I, Archibong A, Udoh J, Nya A, Archibong G. Chronic osteomyelitis of the pubis in the peri-partum period-a rare presentation: case report and a review of the literature. Int J Third World Med. 2008;6:2

9. Dunk RA, Langhoff-Roos J. Osteomyelitis of the pubic symphysis after spontaneous vaginal delivery. BMJ Case Rep. 2010;12:bcr0120102610.

10. Lawford AM, Scott K, Lust K. A case of massive vulvar oedema due to septic pubic symphysitis complicating pregnancy. Aust N Z J Obstet Gynaecol. 2010;50(6):576-7.

11. Froberg $L$, Eckardt $H$. Osteomyelitis of the pubic symphysis - a case report. J Obstet Gynaecol. 2015;35:862-3

12. Brumfield CG, Hauth JC, Andrews WW. Puerperal infection after cesarean delivery: evaluation of a standardized protocol. Am J Obstet Gynecol. 2000; 182(5):1147-51.

13. Chen KT, Huard RC, Della-Latta P, Saiman L. Prevalence of methicillinsensitive and methicillin-resistant Staphylococcus aureus in pregnant women. Obstet Gynecol. 2006;108(3Pt1):482-7.

14. Andrews WW, Schelonka R, Waites K, Stamm A, Cliver SP, Moser S. Genital tract methicillin-resistant Staphylococcus aureus: risk of vertical transmission in pregnant women. Obstet Gynecol. 2008;111(1):113-8.

15. Sakaki H, Nishioka M, Kanda K, Takahashi Y. An investigation of the risk factors for infection with methicillin-resistant Staphylococcus aureus among patients in a neonatal intensive care unit. Am J Infect Control. 2009:37:580-6.

16. Nurjadi D, Klein S, Zimmermann S, Heeg K, Zanger P. Transmission of ST8USA300 Latin American variant methicillin-resistant Staphylococcus aureus on a neonatal intensive care unit: recurrent skin and soft-tissue infections as a marker for epidemic community-associated-MRSA colonization. Infect Control Hosp Epidemiol. 2017;38:883-5.

17. Shen CJ, Wu MS, Lin KH, Lin WL, Chen HC, Wu JY, et al. The use of procalcitonin in the diagnosis of bone and joint infection: a systemic review and meta-analysis. Eur J Clin Microbiol Infect Dis. 2013;32:807-14.

18. Zhao J, Zhang S, Zhang L, Dong X, Li J, Wang Y, Yao Y. Serum procalcitonin levels as a diagnostic marker for septic arthritis: a meta-analysis. Am J Emerg Med. 2017:35(8):1166-71.

19. Paccolat C, Harbarth S, Courvoisier D, Irion O, de Tejada BM. Procalcitonin levels during pregnancy, delivery and postpartum. J Perinat Med. 2011;39(6): 679-83.
20. Karchevsky M, Schweitzer ME, Morrison WB, Parellada JA. MRI findings of septic arthritis and associated osteomyelitis in adults. AJR Am J Roentgenol. 2004;182:119-22.

21. Yan CX, Vautour L, Martin MH. Postpartum sacral insufficiency fractures. Skelet Radiol. 2016:45:413-7.

22. Bierry G, Huang AJ, Chang CY, Torriani M, Bredella MA. MRI findings of treated bacterial septic arthritis. Skelet Radiol. 2012;41:1509-16.

23. Liu C, Bayer A, Cosgrove SE, Daum RS, Fridkin SK, Gorwitz RJ, et al. Clinical practice guidelines by the Infectious Diseases Society of America for the treatment of methicillin-resistant Staphylococcus aureus infections in adults and children. Clin Infect Dis. 2011:1:52.

24. Turner NA, Sharma-Kuinkel BK, Maskarinec SA, Eichenberger EM, Shah PP, Carugati M, et al. Methicillin-resistant Staphylococcus aureus: an overview of basic and clinical research. Nat Rev Microbiol. 2019;8:1.8-55.

\section{Publisher's Note}

Springer Nature remains neutral with regard to jurisdictional claims in published maps and institutional affiliations.
Ready to submit your research? Choose BMC and benefit from:

- fast, convenient online submission

- thorough peer review by experienced researchers in your field

- rapid publication on acceptance

- support for research data, including large and complex data types

- gold Open Access which fosters wider collaboration and increased citations

- maximum visibility for your research: over $100 \mathrm{M}$ website views per year

At BMC, research is always in progress.

Learn more biomedcentral.com/submissions 